\title{
La photographie comme trace
}

\author{
EMMANUEL GARRIGUES \\ Université Paris 7 - Denis Diderot
}

\begin{abstract}
L'essence de la photographie ne peut être (si elle existe) que le Nouveau dont elle a été l'avènement.

ROLAND BARTHES. La chambre claire ${ }^{1}$.

Ce n'est pas sans raison qu'on a comparé les vues d'Atget à celles d'un théâtre du crime. Dans nos villes est-il un seul coin qui ne soit un thêâtre du crime, aucun passant qui ne soit un criminel?.
\end{abstract}

W. BEnjamin. Petite histoire de la photographie ${ }^{2}$.

Depuis que la photographie existe, c'est-à-dire plus d'un siècle et demi, sa définition pose problème. Sous l'apparence de débats techniques, c'est son contenu, le lien fort mais particulier de ce dernier avec la realité, et les effets sur les regardeurs, évoluant avec le temps, qu'entrainent cette difficulté, récurrente. Nous ne ferons pas le tour de différentes approches théoriques depuis le xIXème siecle; mais nous retiendrons que toutes s'accordent à reconnaitre à la photographie une dimension de trace. Trace chimique, phisique, psychique, réelle ou métaphorique, symbolique ou imaginaire, indicielle ou iconique; peu importe, car il y a au moins convergence sur cet aspect de la photographie comme trace. D'ailleurs, beaucoup de ces débats, que nous ne pouvons qu'évoquer ici, sous l'apparence d'une haute technicité conceptuelle, révèlent qu'à la difficulté de definir la photographie s'ajoute tout simplement celle de définir la réalité.

Partant de l'hypothèse que la photographie a été, pour certaines démarches, une ethnographie visuelle (Margaret Mead et Gregory Bateson, E. Curtis ou encore Malinowski), voire une sociologie visuelle (l'oeuvre d'August Sander sur la société allemande de l'entre-deux-guerres), nous

1 R. BARTHES, La chambre claire. Note sur la photographie (Paris: Gallimard, Le Seuil, 1980), p. 15.

2 W. Benjamin, Essais 1. 1922-1934, ed. Denogl-Conthier (Paris: Bibliothèque Médiations, 1983), p. 168. 
avons ouvert cette hypothèse en proposant de l'analyser comme un révélateur épistémologique des sciences humaines, aussi bien métaphoriquement que concrètement, dans un certain nombre de tentatives de construction de la réalité sociale. Les prémices de cette attitude et de cette démarche méthodologique, on peut les trouver ainsi chez Philippe Ariès quand il interroge autant les documents écrits que les documents iconiques (peintures, enluminures mais aussi photographies comme contribuant à la constitution des représentations et à l'expression des mentalités et des sentiments) et qu'il démontre la démocratisation possible des représentations sociales à travers le passage progressif de la peinture de cour et de la galerie d'ancêtres vers la lente constitution de recueils de daguerréotypes et de photographies qu' vont amorcer les albums de famille au cours du xIXème siècle.

On pourrait également citer Gisèle Freund et rappeler l'importance qu'elle accordait à la photographie dans certaines prises de conscience de groupe et d'identité de groupes, de classes sociales et de milieux, depuis la Commune de Paris jusqu'à la Révolution de 1917 ou jusq'aux congés payés; et du coup le rôle qu'elle a pu jouer dans certains mouvements sociaux, y compris récents, comme on va voir.

Certaines prises de conscience, individuelles ou collectives, provoquées par la photographie comme trace, vont nous permettre de distinguer différentes natures de cette trace et nous aider à cerner en quoi et de quoi il y a trace. Traces scientifiques, traces policières, traces esthétiques, l'éventail est assez ouvert, surtout maintenant; il y a trace parce qu'il y a mémoire: mémoires individuelles et mémoires collectives. Rien n'est plus difficile à analyser que la mémoire, particulièrement dans sa dimension sociale et collective; et quels rôles y jouent les images, les photographies, et la dimension visuelle de la pensée, la pensée visuelle.

\section{LA PENSÉE VISUELLE}

Nous parlons de pensée visuelle parce que nous pensons qu'il y a une dimension visuelle de la pensée; l'etude approfondie de la photographie, depuis une dizaine d'années, nous a, en effet, amené ì formuler cette hypothèse à partir d'une réflexion sur la mémoire et le ssurenir. Mais souvenons-nous que la psychanalyse, des le début du siècle, a montré que le psychisme fonctionne en terme d'associations d'idées et d'images. Nous pensons que la photographie peut contribuer à préciser le fonctionnement mental en terme d'associations d'images. Ce que la photographie apporte comme trace psychique et sociale, comme trace d'une mé- 
moire individuelle et collective, W. Benjamin en a une intuition forte, que nous allons tenter de développer, et qu'il exprime ainsi:

Oui, certes, une autre nature parle à l'appareil photographique en tant qu'elle parle à l'oeil; et sur un autre mode surtout parce qu'à un espace consciemment élaboré par l'homme se substitue un espace où il opère inconsciemment. En général, on se rend compte, par exemple, fut-ce en gros, de la démarche des gens, mais il est clair qu'on ne sait rien de leur attitude pendant cette fraction de seconde où se modifie cette démarche. Or, grâce à ses moyens auxiliaires, ralenti et accéléré, la photographie révèle une telle attitude. Elle seule nous renseigne sur cet inconscient de la vue ${ }^{3}$, comme la psychanalyse sur l'inconscient des pulsions.

En fait, ce n'est pas tellement le ralenti ou l'accéléré qui permettent de voir ce qu'on ne peut pas voir habituellement que "l'instantanéisation" (et sa fixation) d'une fraction de seconde d'un comportement saisi sur le vif et de façon inconsciente. De même, il n'y a pas un inconscient de la vue et un inconscient des pulsions, mais un seul et même inconscient dont la photographie est capable d'enregistrer des traces, en instantané. L'intérêt de la citation, c'est cette notion de l'inconscient de la vue. Il y a longtemps que la prise de vue et l'examen attentif d'une multitude de photographies nous ont appris qu'il y avait un inconscient de la vue, ou encore que l'inconscient est autant constitué de mots que d'images; ce qui confirme l'intuition de W. Benjamin. De plus, cette expression peut englober aussi bien l'inconscient du photographe que celui du regardeur. C'est pourquoi nous n'hésitons pas à parler de pensée visuelle; il y a une dimension visuelle de la pensée d'une part, et comme il n'y a pas de pensée sans langage, comme l'a longuement démontré Merleau-Ponty dans la Phénoménologie de la perception, il y a également communication, consciente et inconsciente, par l'intermédiaire de ce langage qu'est la photographie et qui constitue une part importante de la pensée visuelle, d'autre part.

Ce qui rend la recherche sur la photographie passionnante, c'est sa dimension possible de matérialisation de traces de la pensée visuelle. La photographie a inscrit un regard, une vision (avec sa partie inconsciente), du photographe comme regardeur; elle a cette dimension intime et secrète à laquelle peu pensent. Puis elle va être vu par d'autres regardeurs qui, sans y penser consciemment, vont plus ou moins se projeter (cela dépend de l'intérêt que telle ou telle photographie provoque; nous renvoyons à la notion de punctum proposée par Barthes ${ }^{4}$ pour cet aspect

3 W. Benjamin, "Petite histoire de la photographie", Essais 1. 1922-1934, (Paris: Bibliothèque Médiations, 1983), p. 153. Souligné par moi.

4 R. BARTHES, Ibid., pp. 49 y ss. 
de la question), et s'embarquer dans un jeu d'associations, guidés à leur insu par leur inconscient de regardeur.

C'est à partir de ce genre de réflexion que l'on peut approfondir la nature de trace de la photographie, et comprendre ce qu'elle peut apporter à l'analyse de la mémoire comme mécanisme. Halbwachs a montré à quel point le passé est une reconstruction. P. Bourdieu, de son côté, a montré que l'usage le plus courant de la photo est la constitution domestique d'emblèmes domestiques. Nous avons tenté, dans leur sillage, d'approfondir cette approche de la photo comme trace de la mémoire, à partir de témoignages sur les photos de famille, pour pouvoir pénétrer un tant soit peu dans le contenu lui-même de la photographie: ce qu'elle dit de ce qu'elle ne dit pas, ce qu'on voit de ce qui n'est pas montré.

\section{LA MÉMOIRE DE LA PHOTOGRAPHIE}

W. Benjamin trouvait qu'on s'était trop interrogé sur la photographie comme art et pas assez sur l'art comme photographie; à sa suite, on voudrait s'interroger sur la photographie comme mémoire, car c'est une de ses caractéristiques de base, voire une de ses définitions possible, mais il nous parait important aussi, et sans jouer sur les mots, de comprendre comment la mémoire fonctionne comme photographie, comme images fixées de moments précis, qu'on enfouie, et qui ressurgissent de façon inopinée, sans prévenir, à l'occasion d'une association imprévue de souvenirs, par exemple. C'est ce qui peut se passer quand on consulte son ou ses albums de photo de famille. Sur la base de cette hypothèse, nous avons constitué un numéro spécial de L'Ethnographie ${ }^{5}$. Il rend compte d'une enquête empirique que nous avons menée auprès de plus de 600 personnes et à plusieurs reprises, à propos de leurs albums de famille. Nous allons tenter d'approfondir ici la réflexion sur les liens entre la photographie comme trace et la mémoire, à partir des témoignages que nous avons recueillis.

Disons le tout de suite, chacun des témoignages est, à la fois, unique, précis, et intime. Mais une approche sociologique de l'ensemble, conçu comme un corpus particulier, spécifique et collectif, nous permet de tenter d'accéder à ce que la sociologie attend, c'est-à-dire des amorces de tendances et d'attitudes collectives, et la dimension de déterminisme collectif du contenu des réponses, les tendances communes latentes et permanentes.

\footnotetext{
" L'Ethnographie, 120 (1997). Numéro especial. "Famille et photographie".
} 
Tout d'abord, point important pour la recherche, plus on développe une analyse de la photographie, plus elle apparait comme une matrice de recherches. Sa reproductibilité possible avait amené W. Benjamin à penser qu'elle bouleversait le statut de l'oeuvre d'art, des autres oeuvres d'art quand elles sont photographiées, faudrat-il préciser, car la photo comme oeuvre d'art n'est ni moins ni plus reproductible qu'une gravure: chaque tirage garde sa spécificité et donc son originalité. Cette piste de recherche est loin d'être close, évidemment.

Il y a un lien fort entre famille et photographie, car c'est l'usage le plus courant, comme l'a bien montré P. Bourdieu, mais il faudrait comprendre que ce n'est pas son aspect le plus important, loin de là, même si notre enquête montre aussi qu'amateurs et professionnels ont la même attitude vis-à-vis de leurs photos de famille. Il y a tellement d'autres usages et apports de la photographie qu'il ne faudrait pas s'enfermer dans ce constat déjà daté.

Il y a une dimension intime permanente de l'usage de la photographie qu' rejoint malicieusement, et probablement inconscient, la dimension autobiographique obligée, même si inconsciente, ou refoulée, d'une majorité de recherches en sciences humaines. Qui ne cherche pas à partir de ses passions ou de ses fantasmes pour comprendre l'humain? c'est ce que n'hésitait pas à avouer et à préciser Lévi-Strauss dans Tristes Tropiques. Ce que la photographie permet de cerner, c'est la rétrospectivité de perspectives, la réversibilité possible des regards et des observations. C'est une leçon majeure de P. Bourdieu: les classeurs classés par leurs classifications, les observateurs observables à partir de leurs observations, les preneurs de photos dévoilés non pas tant par ce qu'ils montrent en photo mais par le fait que cela dévoile en parallèle que ce qu'ils prennent en photo en dit autant sur eux que ce qu'ils croient prendre et montrer pendant ce temps là. Ce qui nous a amené à cette nouvelle appréciation de la photo qui consiste à en faire un révélateur épistémologique possible des sciences humaines dans leur capacité à observer et analyser la réalité sociale.

La photo arrache irréversiblement quelque chose de la réalité et la garde en mémoire, à la disposition d'éventuels regardeurs disponibles. D'ailleurs, l'appareil de photos et la prise de vues apparaissent eux-mêmes et rapidement dans la pratique du terrain, comme ce que le psychanalyste anglais bien connu Winnicot définit comme objets ou espaces transitionnels dans le rapport à l'autrui observé, voire photographié, dans l'observation.

Car la question de fond qui surgit en permanence est toujours la même: peut-on travailler sur l'intime des autres et comment?

Ce que nous apprend l'etude des photographies de famille, c'est l'ancienneté du phénomène et de la pratique qui s'étend sur plusieurs dé- 
cennies et qui remonte jusqu'aux années 1850 , et donc la nécessité d'utiliser (de façon "foucaldienne", les fameuses boites à outils théoriques) l'histoire, la sociologie, l'ethnographie et la psychanalyse —on voit mal d'ailleurs comment la sociologie qui se propose, dès son origine, d'être la synthèse des sciences humaines, pourrait se dispenser de ces différents apportset cela d'autant plus qu'il ne s'agit pas tant de repérer les usages sociaux de la photo que de pénétrer enfin leur contenu, c'est-à-dire ce qu'elles disent de ceux qui les ont fixé, et surtout ce qu'elles disent à ceux qui les ont gardé et à qu'il arrive de les regarder, mais aussi ce que cela leur fait, et comment ils en parlent. Il y a là un problème de fond que la sociologie n'a pas pu encore étudier: le contenu des photographies change constamment avec le temps, ce qu'entraine la transformation incessante du sens du contenu autant pour les photographiés que pour les regardeurs. L'étude de l'évolution du contenu, dans son importance sociale, nous apparait infiniment plus féconde et révélatrice que l'étude du simple usage de la pratique. Comment approcher autrement les multiples liens sous-jacents et inconscients que les photos de famille provoquent ou qu'entretiennent avec elles ceux qui les détiennent.

Dernier point méthodologique, qui se révèle un champ entier d'étude spécifique: la photo comme prétexte à entretien et à expression: la photo fait parler, fait écrire; elle pousse à l'expression et peut devenir, sur le plan méthodologique, une technique d'entretien. Elle est aussi un champ entier de déclenchement d'écriture. Nous ne pouvons ici qu'évoquer rapidement ce registre immense de recherche entre la photographie et l'écriture. N'en donnons qu'un seul exemple: Brassaï, photographe et écrivain, a longuement travaillé sur l'oeuvre de Proust. Il montre, dans une étude publiée récemment ${ }^{6}$, qu'il y a une relation forte entre la photographie et l'art même de Proust. Non seulement Proust était un collectionneur passionné de photographies, mais encore sa technique narrative des changements de perspective, d'angles d'optique et de cadrages, sans parler de certaines photographies dans le cours du récit, semblent directement influencées par la photographie. Et surtout Brassaï rapproche la "mémoire involontaire" de "l'image latente" telle qu'elle apparait dans le bain du révélateur. Proust lui-même fait de la photographie une métaphore de la mémoire involontaire. C'est dans la photographie, née elle aussi du désir d'arrêter l'instant, pour le fixer à jamais dans une sorte d'éternité, que Proust a trouvé sa meilleure alliée. C'est ce que ne cessent de répéter nos correspondants dans

6 Brassaï, Marcel Proust sous l'emprise de la photographie (Paris: Gallimard, 1997). Enfin certains livres de C. Simon, M. Duras, H. Guibert, etc., sortent directement de souvenirs photographiques ou de photographies. 
leur incessant travail de deuil entretenu par la contemplation ponctuelle, voire hasardeuse, de leurs albums et photos de famille.

\section{LA PHOTO DE FAMILLE COMME TRACE}

La sociologie a montré, il y a une trentaine d'années, que l'usage le plus fréquent de la photo, c'était cette construction officielle d'une représentation officielle des moments forts de la famille. Construction officielle parce qu'elle enregistre, non les moments forts de la vie de famille, mais leur mise en scène officielle. Ce n'est pas les mariés au moment du mariage pris en instantané, c'est le moment où ils se fabriquent eux-mêmes une position officielle qui donne lieu à ce qu'on peut appeler une photo officielle, contrairement à une prise de vue instantanée et improvisée qu' correspondrait mieux à l'essence de la photo. C'est pourquoi P. Bourdieu parlait de fabrication domestique d'emblèmes domestiques. Et, à ce moment là, en effet, la photographie, dans cet usage domestique et mineur, très restrictif par rapport à ses véritables possibilités, contribuait, à son insu, à une idéalisation de la famille.

Les témoignages de nos correspondants nous ont vite montré qu'il ne s'agissait que de la partie manifeste du processus, et non de son importance latente et permanente. Loin d'idéaliser la famille, les albums constitués lentement et sûrement, consultés et complétés à l'occasion, contribuent et de façon incessante, à un travail permanent d'entretien de la mémoire auprès des photographiés et de leurs descendants, dont l'aspect le plus aigu et le plus récurrent, le plus douloureux, le plus dur à vivre et à exprimer, est un interminable travail de deuil.

Roland Barthes, dans son célèbre essai sur la photographie en est une illustration exemplaire. Il cherche à définir l'essence de la photographie à partir d'une photographie de sa mère qui vient de mourir. Travail étonnant qui marie à la fois une recherche scientifique mais qui se révèle être, en même temps et pleinement, un travail de deuil. Il montre aussi à quel point la photographie appelle l'écriture, et comment elle peut exprimer à le fois de la science et de l'art.

Mais ce qu'apportent de plus précis les témoignages sur les photos de famille, c'est une série de mécanismes, conscients et inconscients, qui montre à l'oeuvre l'incessant cheminement de la mémoire et qui dévoile partiellement quelques aspects du fonctionnement visuel de la pensée.

Ainsi, la photographie, en bousculant et télescopant les temps et les temporalités, dans les usages trop fréquents, hâtifs et maladroits (pour ne pas dire ignorants) qui en sont faits, contribue alors, elle aussi, à la dis- 
solution des points de repère: hiers vus et lus aujourd'hui, transformant l'hier en un aujourd'hui incertain et troublant, et permettant donc de transformer constamment certains hiers choisis en des aujourd'huis de plus en plus incompréhensibles, tous ces télescopages de temporalité n'étant indéfiniment que des séquelles d'un travail de deuil qui ne s'avoue jamais complètement comme tel, tout en continuant à travailler inconsciemment les regardeurs.

Ce travail d'aller-et-retour entre le présent et le passé a sa contrepartie également. Si derrière l'envie d'idéaliser sa famille à travers ses albums, se cache le secret permanent travail de deuil, c'est parce que les albums servent aussi de garde-fous de la mémoire. Ils sont des rappels, cruels parfois de réalités plus ou moins oubliées et enfouies. A ce moment-là la photographie fonctionne comme un accroche-mémoire, comme une madeleine de Proust; plus qu'un dépot solennel du souvenir et de la mémoire, elle devient, de façon dynamique, un lieu d'animation, de ressourcement, de recréation de la mémoire et même de recréation active et permanente de cette mémoire.

Ce qui permet de comprendre que la mémoire est une dynamique, constamment active, et non une statique. La photographie est bien, alors, une mémoire des absents et du passé; elle est même un des médias privilegiés de la mémoire. Mais la mémoire nous joue constamment des tours, entre ce qu'elle ne dit pas de ce qu'elle dit, et entre ce qu'elle montre de ce qu'elle ne montre pas, comme la photo.

Un des aspects les plus essentiels, c'est que la photographie provoque et évoque autant la présence que l'absence. Ce jeu sur la présence-absence permet de mieux saisir le processus actif de la pensée visuelle. Par exemple, un de nos correspondants nous adresse la photo d'un lac. C'était le lieu de vacances de son grand-père et d'un de ses amis. Dans son album de famille, il choisit cette photo, car pour lui, elle représente son grand-père, mais il ne réalise pas que le regardeur ne voit qu'un lac pendant que lui y voit son grand-père. C'est ce télescopage de vision qui nous incite à parler de pensée visuelle. "Pendant que je te dis ça, je vois ça, et ce que je vois, c'est justement ce que je te dis". La photo comme mémoire du passé parle des absents dans le présent du regardeur; elle joue comme une métonymie: un détail arrache la signification, l'emporte, l'impose. A ce moment là, elle peut montrer autre chose que ce qu'elle représente.

W. Benjamin a bien vu ce jeu des différentes temporalités, déjà évoqué, quand il parle de la photo comme "le lieu imperceptible où, dans la façon d'être singulière de cette minute depuis longtemps révolue, niche encore aujourd'hui l'avenir, et si éloquent que, par un regard rétrospectif, 


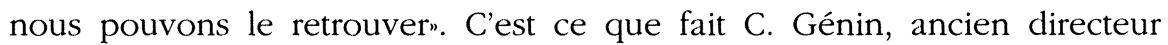
de la communication de Kodak-Pathé, quand il commente ainsi l'envoi d'une photo de ses trois enfants: “Icône familiale. Evocation du bonheur. Garder une trace. Quoiqu'il arrive cette image correspondra toujours à la réalité de cet instantw. Notre enquête demandait qu'on envoie les photos préférées et privilégiées choisies dans les albums et photos de famille et qu'on explique les raisons de cette élection. Evidemment n'ont répondu que ceux qu'acceptaient de témoigner, et de voir publier leurs photos choisies et leur témoignage. Les autres, ou n'ont pas répondu, ou ont precisé qu'ils ne voulaient pas répondre car ils trouvaient la demande trop indiscrète. Ceux qui ont répondu ont alors été pris dans tout un jeu d'esquive entre l'envie de témoigner et d'être publié et la peur d'en dire trop ou de trop se dévoiler. On voit très vite, sur l'ensemble des réponses, qu'il est plus facile de communiquer une photo que d'en parler, ou encore - ce qui peut être paradoxal dans certains cas- que la photo est perçue comme moins indiscrète que le discours; ce qui n'est pas sûr du tout. Faux aveux ou aveu qui cachent, semi-révélations et semi-confidences, beaucoup de témoignages vont bien au-delà de ce qu'ils semblent dire, car la retenue dans l'expression, conjuguée à la photographie, devient soudainement bien plus expressive que le discours qui cherche à ne pas trop en dire. Là façon de ne pas trop en dire en dit plus que ce qui est dit, pendant que la photo montre.

Mais ce qui est vrai du texte l'est également des photos. Elles montrent beaucoup plus que ce qu'elles montrent. Ainsi, un mari envoie un portrait à contre-jour de sa femme, accompagné d'une déclaration de fidélité amoureuse. On voit à peine un cinquième du visage qui se dissout dans l'obscurité, et du coup on regarde plus que si on voyait. On constate alors que les tactiques pour ne pas en dire trop en disent plus en elles mêmes que ce qu'elles tentent de dissimuler. La psychanalyse parlerait d'ambivalence des sentiments, et, sans abuser de son recours, on voit aussi tout un jeu d'identifications et de projections, que les textes dévoilent dans les photos. Il est facile de constater combien de liens oedipiens forts, tel Barthes travaillant sur la photo de sa mère, se montrent, en ne se disant qu'à mi-voix; car ce n'est pas par hasard que les pères ont tendance à envoyer des photos de leur fille, les mères, de leur fils, et aussi les fils de leur mère et les filles de leur père. Une jeune femme envoie une photo d'elle, enfant, prise par son père, mort entre temps: chaque fois qu'elle se regarde sur cette photo, qui est un regard "fixé" de son père sur elle, elle peut, en même temps et discrètement, s'identifier à lui, ou encore le voir, lui, pendant qu'elle croit qu'on ne voit qu'elle, car finalement, il est là aussi, dans la photo, même si ça n'a pas l'air de se voir. 
On pourrait parler du jeu des regards, yeux dans yeux, quand le photographié fixe l'objectif et donc, son regardeur. C'est le cas de ce jeune homme qui pleure chaque fois qu'il se voit regardé par sa mère, de façon "résurrectionnelle" (R. Barthes), dans ce portrait qu'il a fait d'elle, à sa demande à elle et pour lui, peu avant qu'elle ne meurt d'un cancer. Indéfiniment, elle est morte et elle va mourir, et elle le regarde tristement dans les yeux, dès qu'il jette un coup d'oeil sur cette photo. Il suffit même qu'il voit cette photo en souvenir, car il doit aussi se souvenir des moments qui ont précedé et suivi cette prise de vue. Et ces souvenirs là, avec images, peuvent ressurgir à tous moments.

La photo est, à la fois, une mémoire et un miroir; elle fonctionne bien souvent comme un test projectif, mais surtout comme matérialisation extérieure et arbitraire d'images mentales, qu'elle contribue également à créer.

Trace mentale et sociale, elle a de multiples aspects encore qu'on ne peut développer ici. R. Depardon a bien montré, après la mort de Lady $\mathrm{Di}$, que la photographie était coincée socialement, politiquement et idéologiquement, entre le vol apparent de l'instantané et la falsification. Elle a servi aussi à identifier et à retrouver, grace aux photographes associés à des mouvements humanitaires, des milliers d'enfants abandonnés par leurs parents dans leur fuite éperdue des massacres du Rwanda. Traces politiques, policières, et autres. Traces artistiques qui nécessiteraient une autre étude, à part entière.

Les photos de famille, pour s'en tenir à elles, dans cette étude, dévoilent cette attitude ambivalente déjà observée du privé rendu public et du social intériorisé de façon tellement répétitive qu'il devient le privé d'une majorité de gens, avec une dialectique permanente du dévoilement et du secret. Traces mentales matérialisées, elle peuvent aider à comprendre comment fonctionne la mémoire et la dimension visuelle de la pensée.

Le poète Pessoa l'exprime très simplement dans son Livre de l'intranquillité: "Je vogue, l'attention concentrée tou entière dans mes sens, sans pensée, ni émotion. Je me suis éveillé tôt; je suis descendu dans la rue sans préjugé. J'examine comme un qui songe. Je vois comme l'on pense."

\section{LA PHOTOGRAPHIE COMME ÉLÉMENT DE BASE DE LA CONSTRUCTION D'UNE MÉMOIRE COLLECTIVE. LES VILLAGES NOIRS EN FRANCE ET EN EUROPE}

Dans toute cette étude, notre fil conducteur était de considérer la photographie comme trace qui permet de comprendre comment fonctionne la mémoire et la pensée visuelle. La découverte et la rencontre, grâce à Gérard Lévy, de son exceptionnelle collection de cartes postales ancien- 


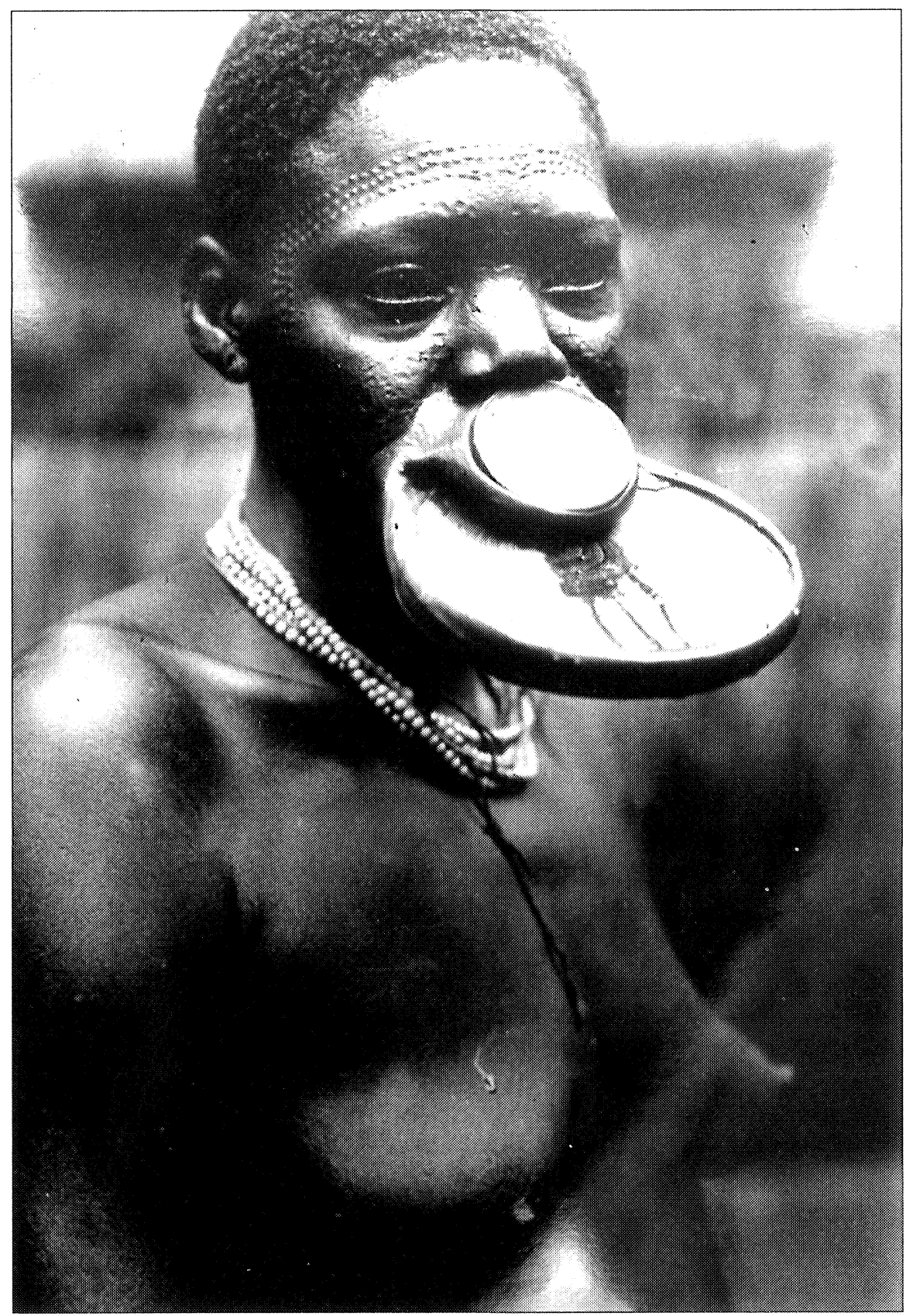

Fig. 1.-Collection Gérard Lévy. Paris. Jardin d'acclimatation, 25-VI-1930. 
nes, montrant photographiquement le phénomène des "villages noirs" en France et en Europe, entre 1877 et 1930, nous imposent soudainement d'élargir encore notre propos dans sa dimension sociologique et historique. Cette collection permet de comprendre la difficulté qu'ont les sociétés à accepter et à assumer les moments les plus sombres de leur histoire, comme elle aide à préciser comment peut se constituer, à travers les vicissitudes sociologiques et idéologiques, le savoir du regard des individus. Le regard est lié au bagage culturel du regardeur; son acuité et son intelligence de ce qu'il voit sont liées à ce bagage.

Gérard Lévy, en nombre d'années d'une patiente recherche, a accumulé plusieurs milliers de photographies, montrant des exhibitions de villages noirs, en France et en Europe pendant plus d'un demi-siècle. Le phénomène est une expression d'un moment particulièrement extensif de la colonisation par les nations d'Europe en voie d'industrialisation accélérée d'un certain nombre de pays d'Afrique et d'Asie à la fin du XIXème siècle. Ainsi, la France et l'Angleterre, comme l'Allemagne et la Hollande, avaient leurs empires, en Afrique en particulier. Les expositions universelles de la fin du xixème siècle étaient l'occasion de faire connaitre dans ces pays d'Europe les productions de leurs colonies. Parmi tous ces produits importés et exhibés, l'un d'entre eux pouvait provoquer une curiosité humaine particulièrement puissante: l'homme de ces contrées lointaines et sauvages car non civilisées.

G. Collomb montre bien l'évolution de la construction du regard à l'égard des "sauvages" dans la présentation des photographies de Roland Bonaparte exposées au Musée de l'Homme à Paris en $1992^{7}$ :

A l'image du bon sauvage installé dans un confortable état de nature, que le XviIIème siècle des philosophes avait tant célébrée, le xıxème siècle positiviste va opposer celle du sauvage, primitif ou déchu, que la science anthropologique naissante entreprendra de situer sur une échelle de l'humanité... Construction théorique d'une représentation hiérarchisée de l'humanité et politiques coloniales se conjuguent, se renforçant et se légitimant l'une l'autre.

C'est bien ce que l'on constate en regardant ces photographies montrant ces exhibitions d'humains à l'occasion d'expositions universelles d'abord, puis d'expositions coloniales, puis progressivement d'expositions exotiques de toutes sortes, à Paris et en province, en France et dans les pays voisins et consistant essentiellement à faire de la monstration de ces humains primitifis bizarres un des clous (au sens du stigmate) de la chose.

7 G. Collomb, "La construction d'un regard", en Kalina: Des amérindiens à Paris. Exposition des photographies du prince Roland Bonaparte au Musée de L'Homme (Paris: Créaphis, 1992), p. 19. 


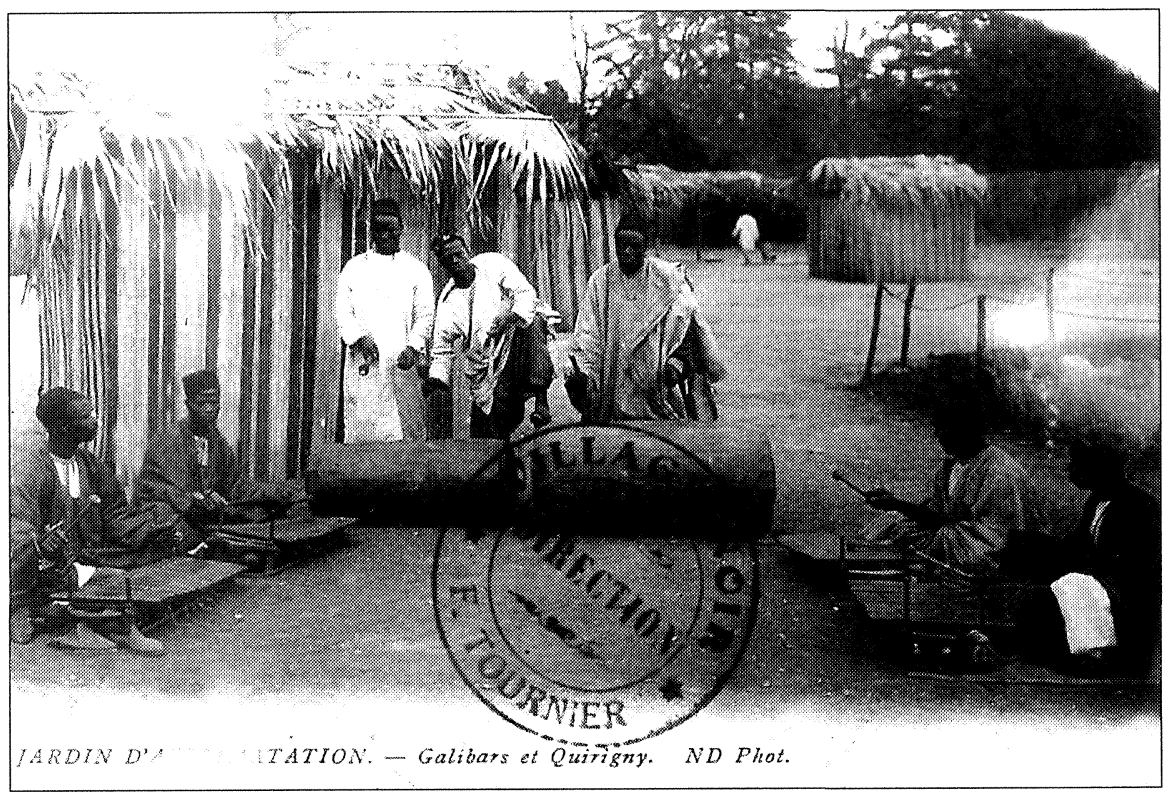

FIG. 2.-Collection Gérard Lévy. Paris. 1912.

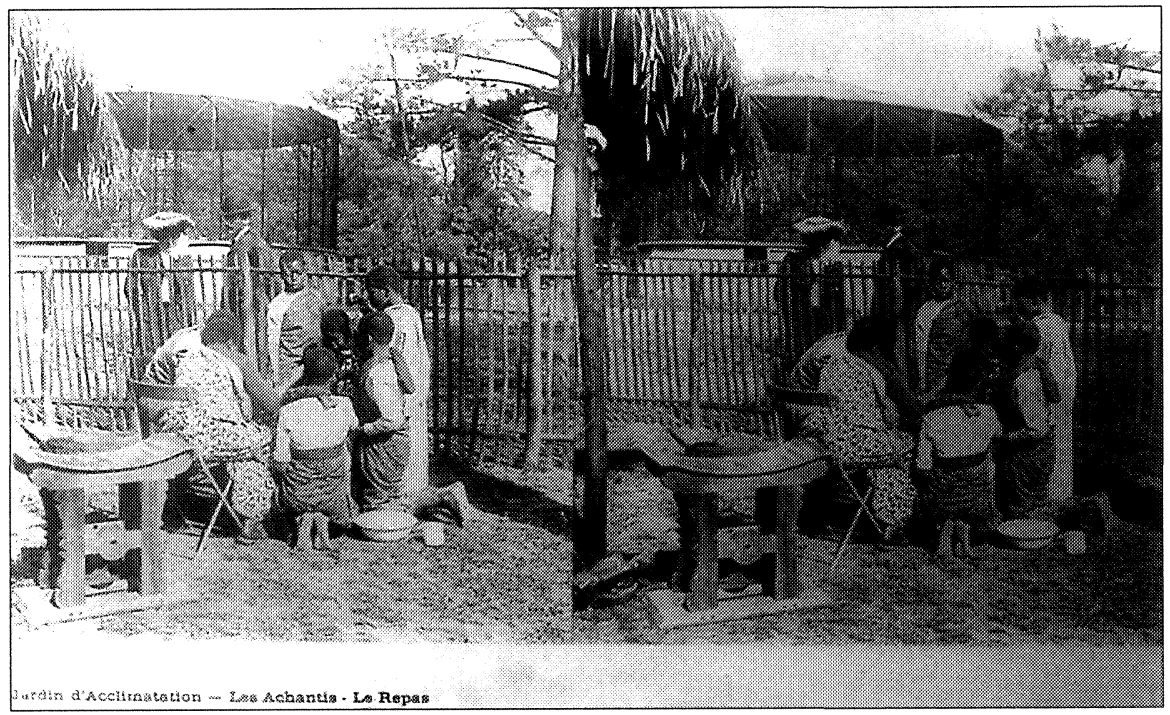

FIG. 3.-Collection Gérard Lévy. Paris. 1906. 
L'ignominie de cette chose, qu'on ne peut peut-être apprécier que maintenant, dans une histoire rétrospective du regard, impose une rigueur éthique dans l'analyse du phénomène. Elle permet de comprendre, en effet, un instant particulier et particulièrement long, de l'archéologie de la construction de l'anthropologie comme science humaine, et nous insistons volontairement sur ce qualíficatif d'humain, pour rappeler qu'il s'agit du lent passage d'une anthropologie physique naturaliste (on mesure les cranes) à une anthropologie sociale et culturelle (on étudie les moeurs sans les juger moralement).

Roland Bonaparte photographie les indiens d'Amérique du Nord au Jardin d'acclimatation à Paris, avant d'avoir l'idée de les photographier chez eux. Le Jardin d'acclimatation, creé au xIxème siècle, est destiné à acclimater à Paris des plantes, des animaux, voire des "hommes", pourquoi pas. Il faut voir les sauvages ici, à Paris, avant de s'acclimater, lentement mais sûrement, à l'idée de les observer chez eux. Lent trajet de la science de l'homme et de la considération de l'homme par l'homme dans toutes ses variations humaines possibles. On voit bien qu'on peut observer à travers ces milliers de cartes postales sur plus d'un demi siècle la façon dont l'homme observe et considère l'homme, aussi bien d'un point de vue scientifique (point de vue savant dont rend compte G. Collomb), que d'un point de vue plus collectif, plus proche de la mémoire collective quotidienne, ce que G. Lévy ne peut s'empêcher d'appeler le "Zoo humain". En effet, la plupart de ces expositions n'hésitaient pas à reconstituer, de façon fantaisiste, les villages, l'habitat, les échoppes, les cases, etc. de tous ces humains bizarres et exotiques; et l'espoir secret des managers professionnels de ces spectacles était qu'une des femmes importées accouche d'un enfant, comme sommet de l'exhibition et de l'attraction, là encore comme clou du spectacle destiné à attirer les visiteurs payants, ou encore le sommet de ce numéro de cirque pour le dire autrement.

Cette collection mérite une étude spécifique à part entière, que nous avons entamée par ailleurs; nous ne pouvions que la présenter brièvement dans cet article. Elle ouvre un champ de recherche spécifique sur l'archéologie de l'anthropologie scientifique contemporaine car elle permet de comprendre mieux que l'objet et le sujet d'une science humaine sont en rapports dialectiques et évoluent en parallèle. En effet, pendant que nous étudions scientifiquement cette collection et, grâce à elle, ce phénomène des "villages noirs", nous contribuons aussi à affiner et préciser la construction scientifique de notre regard.

Nous ne pouvons oublier que les "villages noirs" en France apparaissent à peine, en 1877, une génération après l'abolition "définitive" de l'esclavage en 1848; et no disparaissent que quelques années avant l'apparition des camps 


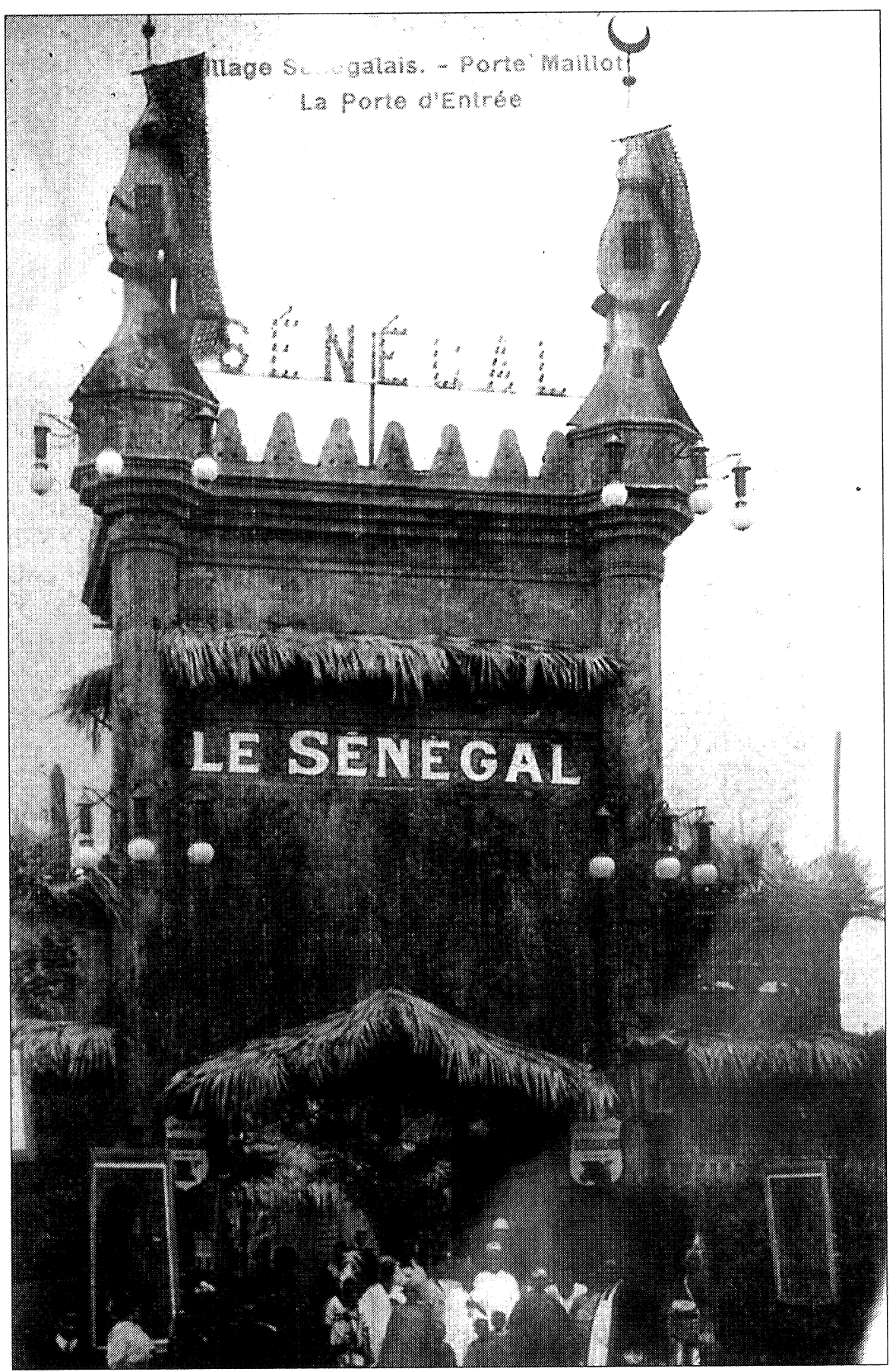

Fig. 4.-Collection Gérard Lévy. Paris. Village Sénégalais. 1908.

H 
de concentration et d'extermination. Ces rapprochements historiques nous paraissent importants pour comprendre un peu comment fonctionnent et évoluent les représentations collectives et le psychisme collectif.

\section{BIBLIOGRAPHIE}

ARIÈs, Ph., 1960: L'enfant et la vie familiale sous l'Ancien Régime, Paris: Plon.

ATGET, 1992: Atget Paris. Bibliothèque Nationale, Mission du Patrimoine photographique, Musée Carnavalet, Institut Français d'architecture. Presentation de Lauro Beaumont-Maillet, Paris: Hazan.

BARTHES, R., 1980: La chambre claire. Note sur la photographie, Paris: Gallimard, Le Seuil, [Cahiers du cinéma].

BEnjamin, W., 1997: "Petite histoire de la photographie"; "L'oeuvre d'art à l'époque de sa reproductibilità technique", en Sur l'art et la photographie, Paris: Arts et Esthétique. - 1983: Essais 1. 1922-1934, Paris: Bibliotèque Médiations.

BOURDIEU, P., 1965: Essai sur les usages sociaux de la photographie, Paris: Minuit.

- 1979: La distinction, critique sociale du jugement, Paris: Minuit.

BRASSAÏ, 1997: Marcel Proust sous l'empire de la photographie, Paris: Gallimard.

Collomb, G., 1992: Kalina: Des Amérindiens à Paris. Exposition des photographies du Prince Roland Bonaparte au Musée de l'Homme, Paris: Créaphis.

COUTANCIER, B., 1902: "Peaux-Rouges". Autour de la collection anthropologique du Prince Roland Bonaparte, Paris: De l'Albaron.

Garrigues, E. (dir.), 1991: "Photographie et Ethnographie», L'Ethnographie, n. 109. [Numéro spécial].

- 1997: "Famille et Photographie", L'Ethnographie, n. ${ }^{\circ}$ 120. [Numéro spécial].

HalbWaChS, M., 1992: Les cadres sociaux de la mémoire, Paris: Albin Michel.

LÉvi-STRauss, C., 1955: Tristes Tropiques, Paris: Plon [Collection Terre Humaine].

PESSOA, F., 1988: Le livre de l'intranquillité, Paris: Christian Bourgois.

Collection de cartes postales de Monsieur Gérard Lévy (inédite), Paris.

Este ensayo propone que el análisis de lo visual se convierta en un auténtico revelador epistemológico de las ciencias sociales. El estudio conjunto de lo etnográfico y de lo fotográfico permite comprender cómo funciona la memoria colectiva, lo que se ejemplifica a través del examen de los llamados "poblados negros" en la Francia del siglo XIX, permitiendo observar el papel legitimador que la imagen tuvo en las políticas coloniales decimonónicas.

The author recommends that the analysis of the visual be turned into an epistemo logical marker for the social sciences. The joint study of ethnographic and photographic documents helps us understand how collective memory works, as an examination of the so-called "black towns" of 19th-century France illustrate. The material on these towns reveals the legitimizing role that images played in the colonial policies of the last century. 